\section{Linking genetics and resistance to infection}

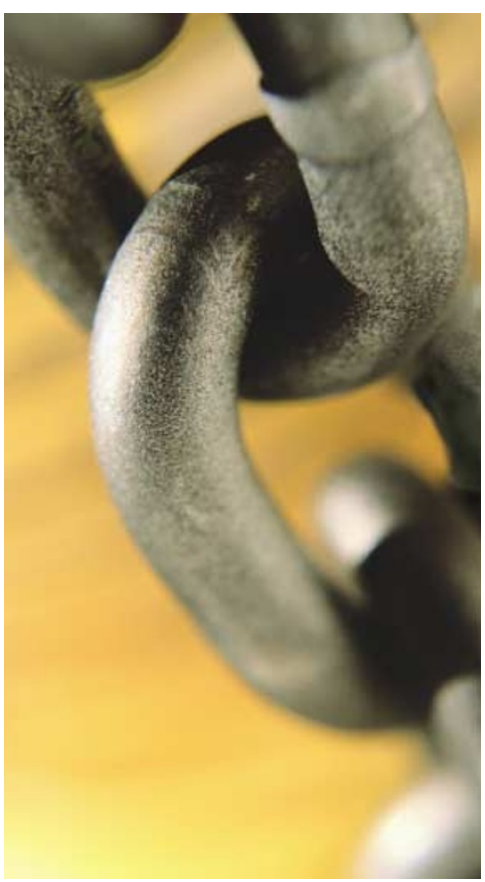

All immunologists know the old adage that MHC polymorphisms are believed to benefit individuals by allowing the presentation of a broad array of antigenic peptides to $\mathrm{T}$ cells. But, direct evidence to support this proposed link between MHC haplotype and resistance to infection has been lacking so far. Now, Janko Nikolich-Ž ugich's lab provides evidence that, in contrast to this widely held view, MHC polymorphisms can influence immune defence by allowing the best $\mathrm{T}$-cell precursors to be selected from a diverse $\mathrm{T}$-cell receptor (TCR) repertoire.

To investigate how MHC polymorphisms influence MHC class-Irestricted $\mathrm{T}$-cell responses, the authors used the mouse strains C57BL/6 (B6; MHC haplotype $\mathrm{H}-2^{\mathrm{b}}$ ) and B6.CH-2bm8 (bm8; MHC haplotype $\left.\mathrm{H}-2^{\mathrm{bm} 8}\right)$, the MHC molecules of which differ by only four amino acids located in the floor of the peptidebinding groove. MHC class I molecules from both strains bind HSV-8p, an immunodominant peptide from glycoprotein B of Herpesvirus hominis type 1 (HVH1).

When challenged with HVH1, bm8 mice were 3-4 times more resistant to infection than were $\mathrm{B} 6$ mice. When $\mathrm{CD}^{+} \mathrm{T}$ cells were depleted from the mice, no differences between the strains were observed in terms of resistance to infection, which indicates that MHC class-Idependent immune responses govern resistance to infection. In (B6 $x$ bm8) $F_{2}$ hybrid mice, a single copy of $\mathrm{H}-2 \mathrm{~K}^{\mathrm{bm} 8}$ conferred enhanced resistance.

In both $\mathrm{B} 6$ and bm 8 mice, $~ 90 \%$ of cytotoxic T lymphocyte (CTL) responses to $\mathrm{HVH} 1$ are specific for the immunodominant peptide HSV8p. Transfer of HSV-8p-specific $\mathrm{T}$ cells from bm8, but not $\mathrm{B} 6$, mice into $\mathrm{F}_{1}$ hybrids conferred significant protection against infection, which indicates that differences in a single allele can enhance protection in a T-cell-dependent manner.
How does this monoallelic difference lead to enhanced protection? Differential peptide binding to MHC molecules was not the answer, because the kinetics of binding of $\mathrm{HSV}-8 \mathrm{p}$ to $\mathrm{H}-2 \mathrm{~K}^{\mathrm{b}}$ and to $\mathrm{H}-2 \mathrm{~K}^{\mathrm{bm} 8}$ were identical. The possibilitity that $\mathrm{H}-2 \mathrm{~K}^{\mathrm{b}}$-mediated negative selection interferes with resistance was ruled out also.

Because $\mathrm{H}-2 \mathrm{~K}^{\mathrm{bm} 8}$ positively selects $\mathrm{CD}^{+} \mathrm{T}$ cells with a broader TCR repertoire than does $\mathrm{H}-2 \mathrm{~K}^{\mathrm{b}}$, the authors next decided to investigate the influence of the TCR repertoire on the quantity and quality of HSV$8 p$-specific CTL responses. No quantitative differences in the numbers of CTL precursors that were mobilized in response to infection were observed between the strains. In B6 mice, the CTL repertoire is dominated by TCRs with two variableregion $(\mathrm{V}) \beta$-chains, $\mathrm{V} \beta 8$ and $\mathrm{V} \beta 10$, whereas bm 8 mice use these $\mathrm{V} \beta$ chains plus five others. The number of $\mathrm{V} \beta 8^{+} \mathrm{T}$ cells is similar in each strain. If the magnitude of the CTL response is an important parameter, depletion of

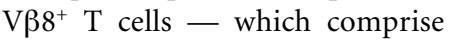
$\sim 15-25 \%$ of the total response to

\title{
Zebrafish give a clearer view
}

Granulomas are organized structures that typically form during mycobacterial infections as a result of complex interactions between mycobacteria and the host. By the time that they are first detectable in mammals, granulomas contain both macrophages and $\mathrm{T}$ cells so a longstanding puzzle has been whether granuloma formation is initiated by innate or adaptive immunity. Now, greater transparency (literally) has been provided by the zebrafish.

The embryos and early swimming larvae of zebrafish are optically transparent, making them an ideal model in which to observe pathogen-host interactions in real time. Adult zebrafish, which have both macrophages and $\mathrm{T}$ cells, can form granulomas in response to infection with Mycobacterium marinum, which is a close relative of the human pathogen
Mycobacterium tuberculosis. But, do granulomas form in zebrafish embryos, at which stage only macrophages are present? Muse Davis and co-workers injected zebrafish embryos intravenously with fluorescently labelled M. marinum then observed them by video microscopy. Three days later, infected macrophages could be seen to have extravasated into the tissues, and they had begun to aggregate squeezing together to form clusters with the typical morphology of granulomas. Heatkilled M. marinum and Salmonella arizonae failed to induce the formation of such granuloma-like structures, which indicates that this process is triggered specifically by mycobacteria-host interactions.

To confirm that these were bona fide granulomas, the authors looked at the expression of M. marinum genes that had been characterized previously as being activated in granulomas (granulomaactivated genes, GAGs) or after infection of cultured macrophages (macrophageactivated genes, MAGs). All four MAGs of $M$. marinum were activated soon after phagocytosis by macrophages, but the three GAGs were activated only once the infected macrophages had aggregated. Therefore, the complex bacteria-host interactions that occur in adult granulomas are mimicked in the embryo.

So, it seems that innate immune factors are sufficient to initiate granuloma formation - which goes against previous studies that cast $\mathrm{T}$ cells in the leading role. This is the first time that pathogen-host interactions have been observed in vivo, as they happen. And this study establishes zebrafish embryos as an illuminating model in which to study granuloma formation. Jennifer Bell

(9) References and links ORIGINAL RESEARCH PAPER Davis, J. M. et al. Real-time visualization of mycobacterium-macrophage interactions leading to initiation of granuloma formation in zebrafish embryos. Immunity 17, 693-702 (2002)

\section{WEB SITE}

Lalita Ramakrishnan's lab:

http://depts. washington.edu/daid/faculty/ramakrishnan.htm 